\title{
COMPACTNESS OF SUBSETS OF TYCHONOFF SETS VIA EXPONENTIAL LAWS
}

\author{
PEDRO MORALES
}

(Received 26 March 1982)

Communicated by J. H. Rubinstein

\begin{abstract}
Using the exponential map. in multifunction context, the paper deduces a system of non-Hausdorff theorems which generalize all known Ascoli theorems for the space of continuous functions and the space of point-compact continuous multifunctions.
\end{abstract}

1980 Mathematics subject classification (Amer. Math. Soc.): 54 D 30, 54 C 60, 54 C 35.

\section{Introduction}

In [18] Noble studied the equivalence between the exponential laws and the Ascoli theorems for the space of continuous functions. This paper uses this approach in the space of point-compact continuous multifunctions. In order to eliminate the Hausdorff restriction, the paper uses the Levine generalization of the closed set [11]. To formulate theorems which may be interpreted either in terms of continuous functions or in terms of point-compact continuous multifunctions, the paper introduces the notion of a Tychonoff set.

All unexplained terminology is defined in [14]. In this paper, the following notations will be used:

(1) $X, Y$ and $Z$ denote topological spaces.

(2) $Y^{m X},\left(Y^{m X}\right)_{0}, \mathcal{C}_{0}(X, Y)$ denote, respectively, the set of all multifunctions on $X$ to $Y$, the set of all point-compact members of $Y^{m X}$ and the set of all continuous members of $\left(Y^{m X}\right)_{0}$.

(3) $P\left\{Y_{x}: x \in X\right\}$ denote the $m$-product of the topological spaces $Y_{x}, x \in X$.

(4) $\tau_{p}, \tau_{c}$ denote, respectively, the pointwise topology and the compact open topology.

() Copyright Australian Mathematical Society 1983 
(5) If $f \in Y^{m X}$ and $B \subseteq Y$, we write $f^{-}(B)=\{x: x \in X$ and $f x \cap B \neq \varnothing\}$, $f^{+}(B)=\{x: x \in X$ and $f x \subseteq B\}$.

\section{Fundamental implications}

An element $f \in Z^{m(X \times Y)}$ determines the function $\tilde{f}: x \rightarrow f(x, \cdot)$ on $X$ to $Z^{m Y}$. The function $\mu: f \rightarrow \tilde{f}$ called the exponential map, is a bijection of $Z^{m(X \times Y)}$ onto $\left(Z^{m Y}\right)^{X}$ such that $\mu\left(\bigodot_{0}(X \times Y, Z)\right) \subseteq\left(\bigodot_{0}(Y, Z)\right)^{X}$. If $\tau$ is a topology on $\bigodot_{0}(Y, Z)$ such that

$$
\mu\left(\bigodot_{0}(X \times Y, Z)\right) \subseteq C\left(X,\left(\bigodot_{0}(Y, Z), \tau\right)\right)
$$

we say that $(X, Y, Z, \tau)$ satisfies the partial exponential law. If $\tau$ is a topology on $\bigodot_{0}(Y, Z)$ such that

$$
\mu^{-1}\left(C\left(X,\left(\bigodot_{0}(Y, Z), \tau\right)\right)\right) \subseteq \bigodot_{0}(X \times Y, Z)
$$

we say that $(X, Y, Z, \tau)$ satisfies the inverse partial exponential law.

Let $F \subseteq Z^{m Y}$. We say that $F$ is pointwise bounded if $\overline{F[y]}$ is compact for every $y \in Y$. A subset $T$ of $Z^{m Y}$ will be called a Tychonoff set if, for every pointwise bounded subset $F$ of $T, P\{\overline{F[y]}: y \in Y\} \cap T$ is $\tau_{p}$-compact. For example, $Z^{Y}$ is a Tychonoff set, by the classical Tychonoff theorem; also $\left(Z^{m Y}\right)_{0}$ is a Tychonoff set, by Corollary 7.6 of [14, page 17].

Following Levine [11, page 90], a subset $A$ of a topological space will be called g-closed if $\bar{A} \subseteq U$ whenever $U$ is an open set containing $A$.

If $\tau$ is a topology on $\mathcal{C}_{0}(Y, Z)$ we say that $(Y, Z, \tau)$ satisfies the Ascoli theorem if, for every Tychonoff subset $T$ of $\left(Z^{m Y}\right)_{0}$, a subset $F$ of $\bigcup_{0}(Y, Z) \cap T$ is $\tau$-compact, provided that

(i) $F$ is $g$-closed,

(ii) $F$ is pointwise bounded, and

(iii) $\tau_{p}$ is jointly continuous on the $\tau_{p}$-closure of $F$ in $T$.

If $\tau$ is a topology on $\bigcup_{0}(Y, Z)$ we say that $(Y, Z, \tau)$ satisfies the converse Ascoli theorem if, for every Tychonoff subset $T$ of $\left(Z^{m Y}\right)_{0}$, every $\tau$-compact subset $F$ of $\mathcal{C}_{0}(Y, Z) \cap T$ satisfies the conditions (i), (ii) and (iii).

2.1. THEOREM. If $(X, Y, Z, \tau)$ satisfies the partial exponential law for every compact space $X$, then $(Y, Z, \tau)$ satisfies the Ascoli theorem.

Proof. Let $T$ be a Tychonoff subset of $\left(Z^{m Y}\right)_{0}$ and let $F$ be a subset of $\mathcal{C}_{0}(Y, Z) \cap T$ satisfying the conditions (i), (ii) and (iii). Let $\bar{F}$ be the $\tau_{p}$-closure of $F$ in $T$. By (iii), $\omega:\left(\bar{F}, \tau_{p}\right) \times Y \rightarrow Z$ is continuous, and, in particular, $\bar{F} \subseteq \mathcal{C}_{0}(Y, Z)$. 
Since $T$ is a Tychonoff set and $F$ is pointwise bounded, by an obvious modification of the proof of Lemma 7.8 of [14, page 18], we can conclude that $\bar{F}$ is $\tau_{p}$-compact. Then, by the hypothesis, $\tilde{\omega}: \bar{F} \rightarrow\left(\mathfrak{C}_{0}(Y, Z), \tau\right)$ is continuous. Since $\tilde{\omega}$ is the inclusion map, $\bar{F}=\tilde{\omega}(\bar{F})$ is $\tau$-compact. But $F \subseteq \bar{F} \subseteq \mathcal{C}_{0}(Y, Z) \cap T$. Then (i) implies, by Theorem 2.9 of [11], that $F$ is $g$-closed in $(\bar{F}, \tau)$. Since $(\bar{F}, \tau)$ is compact, Theorem 3.1 of [11] implies that $F$ is $\tau$-compact.

2.2. THEOREM. Let $Z$ be a regular space and let $\tau$ be a regular topology on $\mathcal{C}_{0}(Y, Z)$. If $(X, Y, Z, \tau)$ satisfies the inverse partial exponential law for every compact space $X$, then $(Y, Z, \tau)$ satisfies the converse Ascoli theorem.

Proof. Let $F$ be a $\tau$-compact subset of $\mathcal{C}_{0}(Y, Z) \cap T$, where $T$ is a Tychonoff subset of $\left(Z^{m Y}\right)_{0}$. Then $F$ is pointwise bounded because, if $y \in Y, F[y]=\operatorname{pr}_{y}(F)$ is compact [3, page 116]. Since $\left(\mathcal{C}_{0}(Y, Z) \cap T, \tau\right)$ is regular, Theorem 3.5 of [11] implies that $F$ is $g$-closed in $\bigcup_{0}(Y, Z) \cap T$.

In order to prove the condition (iii), it suffices, by Theorem 10.1 of [14, page 21 , to show that $F$ satisfies the condition (G). Let $F_{0}$ be a $\tau_{c}$-closed subset of $F$ and let $V$ be an open subset of $Z$. It must be shown that $\bigcap_{f \in F_{0}} f^{-}(V)$ and $\bigcap_{f \in F_{0}} f^{+}(V)$ are open in $Y$. Since the inclusion map $i: F \rightarrow \bigodot_{0}(Y, Z)$ is continuous, by the hypothesis, $\mu^{-1}(i)=\omega: F \times Y \rightarrow Z$ is continuous. This implies, in particular, that $\tau$ is finer than $\tau_{c}$ [21, page 49]. Therefore $F_{0}$ is $\tau$-closed in $F$, hence $\tau$-compact.

Let $y_{0} \in \cap_{f \in F_{0}} f^{-}(V)$ and let $\omega: F_{0} \times Y \rightarrow Z$. Then $F_{0} \times\left\{y_{0}\right\} \subseteq \omega^{-}(V)$. Since $F_{0}$ is compact and $\omega^{-}(V)$ is open, by the theorem of Wallace, there exists a neighbourhood $U$ of $y_{0}$ such that $F_{0} \times U \subseteq \omega^{-}(V)$. Let $y \in U$. For $f \in F_{0}$, fy $\cap V \neq \varnothing$, that is, $y \in \bigcap_{f \in F_{0}} f^{-}(V)$. We have, therefore, $U \subseteq \bigcap_{f \in F_{0}} f^{-}(V)$, showing that $\cap_{f \in F_{0}} f^{-}(V)$ is open. Similarly, we show that $\cap_{f \in F_{0}} f^{+}(V)$ is open.

\section{Ascoli theorems}

Let $F \subseteq Z^{m Y}$. Following [15], we say that $F$ is evenly continuous if, whenever $y \in Y, K$ is a compact subset of $Z$ and $V$ is a neighbourhood of $K$, there exist neighbourhoods $U, W$ of $y, K$, respectively, such that, for all $f \in F, f x \cap W \neq \varnothing$ implies $U \subseteq f^{-}(V)$ and $f x \subseteq W$ implies $U \subseteq f^{+}(V)$.

Let $Z=(Z, \mathcal{Q})$ be a uniform space and let $F \subseteq Z^{m Y}$. Following [22], we say that $F$ is equicontinuous if, for $y \in Y$ and $U \in \mathcal{Q}$, there exists a neighbourhood $V$ of $y$ such that, for all $f \in F, f(V) \subseteq U[f y]$ and $f x \cap U[z] \neq \varnothing$ whenever $(x, z) \in V \times f y$. 
Since $\left(X, Y, Z, \tau_{c}\right)$ satisfies the partial exponential law [14, page 18], we deduce from Theorem 2.1 the following consequence:

3.1. Theorem. $\left(Y, Z, \tau_{c}\right)$ satisfies the Ascoli theorem.

3.2. COROLlaRY. Let $Z$ be a regular space and let $T$ be a Tychonoff subset of $\left(Z^{m Y}\right)_{0}$. A subset $F$ of $\left(\mathcal{e}_{0}(Y, Z) \cap T, \tau_{c}\right)$ is compact if

(a) $F$ is g-closed,

(b) $F$ is pointwise bounded, and

(c) $F$ is evenly continuous.

Proof. By Lemma 4.1 of [15], (c) implies the condition (iii).

3.3. CoROllary. Let $Z$ be a regular space and let $T$ be a Tychonoff subset of $\left(Z^{m Y}\right)_{0}$. A subset $F$ of $\left(\mathcal{C}_{0}(Y, Z) \cap T, \tau_{c}\right)$ is compact if

(a) $F$ is g-closed,

(b) $F$ is pointwise bounded, and

(c) F satisfies the condition $(\mathrm{G})$.

Proof. By Theorem 10.1 of [14, page 21], (c) implies the condition (iii).

3.4. Corollary. Let $Z$ be a uniform space and let $T$ be a Tychonoff subset of $\left(Z^{m Y}\right)_{0}$. A subset $F$ of $\left(\bigodot_{0}(Y, Z) \cap T, \tau_{c}\right)$ is compact if

(a) $F$ is a g-closed,

(b) $F$ is pointwise bounded, and

(c) $F$ is equicontinuous.

Proof. By Lemmas 6 and 7 of Smithson [21, pages 257-258], (c) implies (iii).

Let $Z=(Z, \mathscr{Q})$ be a uniform space and let $c Z$ denote the set of all non-empty compact subsets of $Z$. Following Michael [13, page 153], we define on $c Z$ the uniformity $c$ Q having as base the sets of the form $\left\{\left(K_{1}, K_{2}\right): K_{2} \subseteq \cup_{x \in K_{1}} U[x]\right.$ and $K_{2} \cap U[y] \neq \varnothing$ for all $\left.y \in K_{1}\right\}$, where $U \in \mathcal{Q}$. Since, in the proof of Theorem 3.3 of [13, page 160], the restriction that the members of $c Z$ be closed in $Z$ is not used, we may conclude that $c \mathscr{Q}$ induces the Vietoris or finite topology on $c Z$. Accordingly we can identify $f \in \mathcal{C}_{0}(Y, Z)$ with the function $f^{*}: y \rightarrow f y \in c Z$ to obtain the equation $\mathcal{C}_{0}(Y, Z)=C(Y, c Z)$. This identification being understood, we write $f$ for $f^{*}$. It turns out that the topology of uniform convergence $\tau_{u}$ on $C_{0}(Y, Z)$, as defined by Smithson [22, page 253], is the same as the topology of uniform convergence, in the classical sense, on $C(Y, c Z)$, using the uniformity 
$c \mathcal{Q}$. Consequently, a base for the neighbourhood filter of an element $f \in$ $\left(\mathcal{C}_{0}(Y, Z), \tau_{u}\right)$ consists of all sets of the form $N_{d, \varepsilon}(f)=\{g: d(f(y), g(y))<\varepsilon$ for all $y \in Y\}$, where $\varepsilon>0$ and $d$ is a continuous pseudometric on $(c Z, c \mathscr{Q})$.

A subset of a topological space $X$ is called a zero-set if it is the inverse image of 0 under some continuous real-valued function on $X$ [8, page 53]. We note that if $f$ is a real-valued continuous function on $X$, then $\{x \in X: f(x) \geqslant 0\}$ is a zero-set. Let $X, Y$ be topological spaces and let $\Pi_{X}: X \times Y \rightarrow X$ be the first projection. Then $\Pi_{X}$ is said to be $z$-closed [7] if it maps every zero-set of $X \times Y$ onto a closed subset of $X$.

3.5. Theorem. If $Z$ is a uniform space and $\Pi_{X}: X \times Y \rightarrow X$ is $z$-closed, then $\left(X, Y, Z, \tau_{u}\right)$ satisfies the partial exponential law.

Proof. Let $f \in \mathcal{C}_{0}(X \times Y, Z)$, let $x_{0} \in X$ and let $N_{d, \varepsilon}\left(\tilde{f}\left(x_{0}\right)\right)=\left\{g \in \mathcal{C}_{0}(Y, Z)\right.$ : $d\left(\tilde{f}\left(x_{0}\right)(y), g(y)\right)<\varepsilon$ for all $\left.y \in Y\right\}$, where $\varepsilon>0$ and $d$ is a continuous pseudometric on $(c Z, c \mathcal{Q})$. It must be shown that $\tilde{f}^{-1}\left(N_{d, \varepsilon}\left(\tilde{f}\left(x_{0}\right)\right)\right)$ is a neighbourhood of $x_{0}$. Let $\Gamma_{f}: X \times Y \rightarrow Y \times c Z$ be defined by the formula $\Gamma_{f}(x, y)=(y, f(x, y))$. Since $\Pi_{c Z} \circ \Gamma_{f}=f$ and $\Pi_{Y} \circ \Gamma_{f}$ is the second projection on $X \times Y, \Gamma_{f}$ is continuous. Let $S=\left\{(y, K) \in Y \times Z: d\left(f\left(x_{0}, y\right), K\right) \geqslant \varepsilon\right\}$. Then $\Gamma_{f}^{-1}(S)=\{(x, y) \in$ $\left.X \times Y: \quad d\left(f\left(x_{0}, y\right), f(x, y)\right) \geqslant \varepsilon\right\}, \quad$ so $\Pi_{X}\left(\Gamma_{f}^{-1}(S)\right)=\{x \in X$ : $d\left(f\left(x_{0}, y\right), f(x, y)\right) \geqslant \varepsilon$ for some $\left.y \in Y\right\}$ and therefore $X-\Pi_{X}\left(\Gamma_{f}^{-1}(S)\right)=\{x$ $\in X: d\left(f\left(x_{0}, y\right), f(x, y)\right)<\varepsilon$ for all $\left.y \in Y\right\}=\tilde{f}^{-1}\left(N_{d, \varepsilon}\left(\tilde{f}\left(x_{0}\right)\right)\right)$. Since $\Gamma_{f}^{-1}(S)=$ $\left\{(x, y) \in X \times Y:\left(d \circ\left(\tilde{f}\left(x_{0}\right) \times 1_{c z}\right) \circ \Gamma_{f}\right)(x, y) \geqslant \varepsilon\right\}, \Gamma_{f}^{-1}(S)$ is a zero-set. Then $\Pi_{X}\left(\Gamma_{f}^{-1}(S)\right)$ is a closed set not containing $x_{0}$, so $\tilde{f}^{-1}\left(N_{d, \varepsilon}\left(\tilde{f}\left(x_{0}\right)\right)\right)$ is an open set containing $x_{0}$.

By definition, a topological space $X$ is pseudocompact if every real-valued continuous function on $X$ is bounded [8, page 67]. It is known that the product of a pseudocompact space by a compact space is pseudocompact [16, page 20].

3.6. THEOREM. If $Z$ is a uniform space and $Y$ is a pseudocompact space, then $\left(Y, Z, \tau_{u}\right)$ satisfies the Ascoli theorem.

Proof. This follows from Theorems 2.1, 3.5 and Theorem 2.5 of [18, page 397].

3.7. CoRollary. Let $Y$ be a pseudocompact space, let $Z$ be a uniform space and let $T$ be a Tychonoff subset of $\left(Z^{m Y}\right)_{0}$. A subset $F$ of $\left(\mathcal{C}_{0}(Y, Z) \cap T, \tau_{u}\right)$ is compact if

(a) $F$ is g-closed,

(b) $F$ is pointwise bounded, and

(c) $F$ is equicontinuous. 
PRoof. Same as proof of Corollary 3.4 .

\section{Converse Ascoli theorems}

A topological space $X$ is a $k_{3}$-space if $C_{k}(X, Y)=C(X, Y)$ for every regular space $Y$ [19, page 195]. Thus a $k$-space is a $k_{3}$-space but not conversely. In fact, the product of uncountably many copies of the real line, which is not a $k$-space, is a $k_{3}$-space [19, Theorem $\left.5.6(i)\right]$.

If $X$ is compact, $Y$ is a $k_{3}$-space and $Z$ is regular, then $\left(X, Y, Z, \tau_{c}\right)$ satisfies the inverse partial exponential law [15, Theorem 3.4]. From this fact, Theorem 2.2 and Theorem 2 of Smithson [21, page 48], we deduce the following consequence:

4.1. THEOREM. If $Y$ is a $k_{3}$-space and $Z$ is regular, then $\left(Y, Z, \tau_{c}\right)$ satisfies the converse Ascoli theorem.

4.2. Corollary. Let $Y$ be a $k$-space, let $Z$ be a regular space and let $T$ be a Tychonoff subset of $\left(Z^{m Y}\right)_{0}$. If a subset $F$ of $\left(\mathcal{C}_{0}(Y, Z) \cap T, \tau_{c}\right)$ is compact, then

(a) $F$ is $g$-closed,

(b) $F$ is pointwise bounded, and

(c) $F$ is evenly continuous.

Proof. By (iii), $\omega:\left(F, \tau_{p}\right) \times Y \rightarrow Z$ is continuous, therefore $F=\{\omega(f, \cdot)$ : $f \in F\}$ is evenly continuous [15, Lemma 4.2].

4.3. Corollary. Let $Y$ be a $k_{3}$-space, let $Z$ be a regular space and let $T$ be a Tychonoff subset of $\left(Z^{m Y}\right)_{0}$. If a subset $F$ of $\left(\bigodot_{0}(Y, Z) \cap T, \tau_{c}\right)$ is compact, then

(a) $F$ is g-closed,

(b) $F$ is pointwise bounded, and

(c) $F$ satisfies the condition $(\mathrm{G})$.

Proof. By (iii), $\tau_{p}$ on $F$ is jointly continuous. Then, by Corollary 10.6 of [14, page 23], $F$ satisfies (G).

4.4. CoRollary. Let $Y$ be a $k_{3}$-space, let $Z$ be a uniform space, and let $T$ be a Tychonoff subset of $\left(Z^{m Y}\right)_{0}$. If a subset $F$ of $\left(\bigcup_{0}(Y, Z) \cap T, \tau_{c}\right)$ is compact, then

(a) $F$ is g-closed,

(b) $F$ is pointwise bounded, and

(c) $F$ is equicontinuous. 
Proof. By (iii), $\tau_{p}$ on $F$ is jointly continuous. Then, by Lemma 8 of Smithson [22, page 258], $F$ is equicontinuous.

4.5. THEOREM. If $Z$ is a uniform space, then $\left(X, Y, Z, \tau_{u}\right)$ satisfies the inverse partial exponential law.

Proof. Let $f \in\left(Z^{m(X \times Y)}\right)_{0}$ be such that the function $\tilde{f}: X \rightarrow\left(\mathcal{C}_{0}(Y, Z), \tau_{u}\right)$ is continuous. Let $\left(x_{0}, y_{0}\right) \in X \times Y$ and let $\left\{U_{i}\right\}_{1 \leqslant i \leqslant n}$ be a finite sequence of open subsets of $Z$ such that $f\left(x_{0}, y_{0}\right) \subseteq \cup_{i=1}^{n} U_{i}$ and $f\left(x_{0}, y_{0}\right) \cap U_{i} \neq \varnothing$ for all $i=$ $1, \ldots, n$. It must be shown that $f^{-1}\left(\left\langle U_{1}, \ldots, U_{n}\right\rangle\right)$ is open in $X \times Y$.

Since $f\left(x_{0}, y_{0}\right) \in\left\langle U_{1}, \ldots, U_{n}\right\rangle$, there exists $\varepsilon>0$ and a continuous pseudometric $d$ on $(c Z, c$ Q $)$ such that $\mathscr{B}_{d, \varepsilon}\left(f\left(x_{0}, y_{0}\right)\right)=\left\{K \in c Z: d\left(K, f\left(x_{0}, y_{0}\right)\right)<\varepsilon\right\} \subseteq$ $\left\langle U_{1}, \ldots, U_{n}\right\rangle$. Since $\tilde{f}\left(x_{0}\right)$ is continuous, $W=\tilde{f}\left(x_{0}\right)^{-1}\left(\mathscr{B}_{d, \varepsilon / 2}\left(f\left(x_{0}, y_{0}\right)\right)\right)$ is a neighbourhood of $y_{0}$. Moreover, since $\tilde{f}$ is continuous, $V=\tilde{f}^{-1}\left(N_{d, \boldsymbol{e} / 2}\left(\tilde{f}\left(x_{0}\right)\right)\right)$ is a neighbourhood of $x_{0}$. Then $V \times W$ is a neighbourhood of $\left(x_{0}, y_{0}\right)$ contained in $f^{-1}\left(\left\langle U_{1}, \ldots, U_{n}\right\rangle\right)$. In fact, let $(x, y) \in V \times W$. Then $\tilde{f}(x) \in N_{d, \varepsilon / 2}\left(\tilde{f}\left(x_{0}\right)\right)$ and $f\left(x_{0}\right)(y) \in \mathscr{B}_{d, \varepsilon / 2}\left(f\left(x_{0}, y_{0}\right)\right)$, that is, $d\left(f(x, t), f\left(x_{0}, t\right)\right)<\varepsilon / 2$ for all $t \in Y$ and $d\left(f\left(x_{0}, y\right), f\left(x_{0}, y_{0}\right)\right)<\varepsilon / 2$. So, in particular, $d\left(f(x, y), f\left(x_{0}, y_{0}\right)\right)<\varepsilon$ and therefore $f(x, y) \in \mathscr{B}_{d, \mathrm{e}}\left(f\left(x_{0}, y_{0}\right)\right) \subseteq\left\langle U_{1}, \ldots, U_{n}\right\rangle$.

4.6. THEOREM. If $Z$ is a uniform space, then $\left(Y, Z, \tau_{u}\right)$ satisfies the converse Ascoli theorem.

PROof. This follows from Theorems 2.2 and 4.5.

4.7. COROLlaRY. Let $Z$ be a uniform space and let $T$ be a Tychonoff subset of $\left(Z^{m Y}\right)_{0}$. If a subset $F$ of $\left(\bigodot_{0}(Y, Z) \cap T, \tau_{u}\right)$ is compact, then

(a) $F$ is g-closed,

(b) $F$ is pointwise bounded, and

(c) $F$ is equicontinuous.

PROOF. Same as proof of Corollary 4.4 .

4.8. REMARK. Referring to the equivalence relation on a regular space introduced in [14, page 11], we note that if $F^{*}$ is closed then $F \subseteq \bar{F} \subseteq F^{*}$ and therefore $F$ is $g$-closed; moreover, if $F$ is compact then $F^{*}$ is closed [14, Theorem 4.1].

4.9. Remark. Corollary 3.2 together with Corollary 4.2 is the Theorem 5.1 of [15], which, in the case $T=Z^{Y}$, contains the Ascoli theorem 4.1 of [4, page 635]. This latter generalizes the Ascoli theorems of Kelley-Morse [10, page 236], Bagley-Yang [2, page 704], Noble [18, Corollary 4.4] and Kaul [9, Theorem B]. 
4.10. Remark. If we take $T=\left(Z^{m Y}\right)_{0}$ in Corollaries 3.3 and 4.3 , we obtain a $k_{3}$-space generalization of Theorem 10.10 of [14, pages 23-24], which in turn contains the function Ascoli theorem of Gale [5, page 304] and the multifunction Ascoli theorem of Mancuso [12, page 470].

4.11. Remark. If we take $T=\left(Z^{m Y}\right)_{0}$ in Corollaries 3.4 and 4.4 we obtain, because of Theorem 12.2 of [14, page 28], a $k_{3}$-space generalization of Theorem 12.8 of [14, page 31], which in turn contains the function Ascoli theorems of Arens [1, page 491], Myers [17, pages 497-498] and Bagley-Yang [2, page 705], also the multifunction Ascoli theorem of Smithson [22, page 259].

4.12. Remark. If we take $T=Z^{Y}$, Corollary 3.7 together with Corollary 4.7 generalizes the Ascoli theorem of Noble [18, Corollary 4.3 (i)], which in turn generalizes the Ascoli theorem of Glicksberg [6, page 257].

4.13. REMARK. There has recently appeared another definition of even continuity in the space $\left(Z^{m Y}\right)_{0}[20$, page 14]. Using a suitable modification of the arguments used in the proofs, it can be shown that this "even continuity" has the properties stated in Lemma 4.1, and, with the additional point-compact condition, has the property stated in Lemma 4.2 of [15]. These properties established, we can deduce, with greater generality, the Ascoli theorem 3.1 of [20, page 150] from Theorems 3.1 and 4.1.

\section{References}

[1] R. Arens, 'A topology for spaces of transformations', Ann. of Math. 47 (1946), 480-495.

[2] R. W. Bagley and J. S. Yang, 'On $k$-spaces and function spaces', Proc. Amer. Math. Soc. 17 (1966), 703-705.

[3] C. Berge, Topological spaces (Macmillan Company, New York, 1965).

[4] G. Fox and P. Morales, 'A non-Hausdorff Ascoli theorem for $k_{3}$-spaces', Proc. Amer. Math. Soc. 39 (1973), 633-636.

[5] D. Gale, 'Compact sets of functions, and function rings', Proc. Amer. Math. Soc. 1 (1950), 303-308.

[6] I. Glicksberg, 'Representation of functionals by integrals', Duke Math. J. 19 (1952), 253-282.

[7] A. W. Hager and S. G. Mrówka, 'Compactness and the projection mapping from a product space', Notices Amer. Math. Soc. 12 (1965), 368 (abstract 65T-167).

[8] E. Hewitt, 'Rings of real-valued continuous functions I', Trans. Amer. Math. Soc. 64 (1948), 45-99.

[9] S. K. Kaul, 'Compact subsets in function spaces', Canad. Math. Bull. 12 (1969), 461-466.

[10] J. Kelley, General topology (C. Van Nostrand, New York, 1965).

[11] N. Levine, 'Generalized closed sets in topology', Rend. Circ. Mat. Palermo Ser. (2) 19 (1970), 89-96. 
[12] V. J. Mancuso, 'An Ascoli theorem for multivalued functions', J. Austral. Math. Soc. 12 (1971), 466-472.

[13] E. Michael, 'Topologies on spaces of subsets', Trans. Amer. Math. Soc. 71 (1951), 152-182.

[14] P. Morales, 'Non-Hausdorff Ascoli theory', Dissertationes Math. 119 (1974), 1-37.

[15] P. Morales, 'A non-Hausdorff multifunction Ascoli theorem for $k_{3}$-spaces', Canad. J. Math. 27 (1975), 893-900.

[16] S. G. Mrówka, 'Compactness and product spaces', Colloq. Math. 7 (1959), 19-22.

[17] S. B. Myers, 'Equicontinuous sets of mappings', Ann. of Math. 47 (1946), 496-502.

[18] N. Noble, 'Ascoli theorems and the exponential map', Trans. Amer. Math. Soc. 143 (1969), $393-411$.

[19] N. Noble, 'The continuity of functions on Cartesian products', Trans. Amer. Math. Soc. 149 (1970), 187-198.

[20] H. W. Pu, 'Another Ascoli theorem for multi-valued functions', Bull. Inst. Math. Acad. Sinica 1 (1973), 145-153.

[21] R. E. Smithson, 'Topologies on sets of relations', J. Natur. Sci. and Math. (Lahore) 11 (1971), 43-50.

[22] R. E. Smithson, 'Uniform convergence for multifunctions', Pacific J. Math. 39 (1971), 253-259.

\section{Université de Sherbrooke}

Sherbrooke, Québec, Canada 